\title{
ML-DEs: A program for designing efficient multilevel studies
}

\author{
Wirfried Cools, Wim Van den Noortgate, and Patrick Onghena \\ Katholieke Universiteit Leuven, Leuven, Belgium
}

\begin{abstract}
The multilevel model is increasingly used as a flexible tool in the statistical analysis of dependent behavioral research data. A drawback of this model's flexibility is that it complicates designing the study. For example, an important additional consideration in the design of a multilevel study is choosing the number and the size of the clusters to sample to ensure sufficient efficiency as quantified by precision, bias, or statistical power. To help researchers in designing their multilevel study, a user-friendly simulation tool is introduced (Multilevel Design Efficiency Using Simulation, or ML-DEs), also allowing for design questions that have not been dealt with analytically in the literature, while avoiding complex specifications of simulation studies. ML-DEs generates MLwiN macros for running the simulations and handles its output using $\mathbf{R}$ scripts to compare the designs' efficiencies for both fixed and random parameters, allowing for small sample sizes, unbalanced data, and more than two levels.
\end{abstract}

Various designs of varying efficiency can be used to answer specific research questions. They may differ in many ways: in the amount of information they provide as quantified by, for example, the accuracy of estimation (Kelley \& Maxwell, 2003) and/or the power for statistical testing (J. Cohen, 1988); or alternatively, in how many resources are required to obtain a certain amount of information. Carefully designing studies to be efficient is especially important for behavioral research, which often has to deal with small effects and considerable uncertainty about the estimates (Pillemer, 1984).

Whereas it is well understood how to increase the design efficiency for simple models (Howell, 2005), for more complex models and their corresponding analyses this relation between design and efficiency becomes much less straightforward, as with, for example, multilevel data analyses (Snijders, 2005). The multilevel statistical model is increasingly used, because it elegantly takes into account dependencies among observations that exist because of multistage sampling, with sampled observations embedded within clusters; or because repeated measurements were performed, with a sequence of observations embedded within units. Similarly, meta-analyses and multivariate analyses can also be dealt with using multilevel analyses (Raudenbush, 1988; Van den Noortgate \& Onghena, 2003, 2006).

Multilevel design efficiency has already been studied analytically, as well as in simulation studies. Whereas the analytical studies only deal with quite simple models and imply several assumptions, the results of the simulation studies may be difficult to generalize about, because data are generated from a specific model and specific parameter values. In response to both problems, this article will present a user-friendly tool named Multilevel Design Efficiency Using Simulation (ML-DEs), which allows applied researchers to derive more efficient multilevel designs for their own research. Unlike existing programs that use analytical results, this tool will allow for dealing with small sample sizes at any of the levels, unbalanced data, more than just two levels, and tests for both the fixed and the random parameters. Because simulations set up with ML-DEs are conditional on alternative models of interest, there is no need for generalization, as there would be when using simulation studies found in the literature.

A specific focus in the present article will be on the optimal number of clusters and/or groups to sample at higher levels in order to provide maximum information on the parameters of interest, reflected in a more efficient estimation and testing of both the regression coefficients that refer to the fixed part and the (co)variance parameters that refer to the random part. First, the relevant parameters for design efficiency are introduced; second, the multilevel model will be discussed briefly in relation to design efficiency; third, the exploration of design efficiency is commented on; finally, our program ML-DEs is highlighted and illustrated by means of an example.

\section{DESIGN EFFICIENCY}

As briefly mentioned in the introduction, design efficiency reflects the amount of information gained from a study in relation to the resources required for it. This information can be quantified from the perspective of estimating an effect or from the perspective of testing a hypothesized effect, depending on the specific research questions of interest (Kelley \& Maxwell, 2003). 
To introduce design efficiency, we start from a simple situation. Suppose that a linear regression with a single predictor, $\mathbf{x}$, that takes on the predictor values -0.5 and 0.5 , is used to compare the mean performance of two groups. Each observation could then be equated to a general mean and a group-dependent difference from that mean:

$$
y_{i}=\beta_{0}+\beta_{1} x+e_{i} \text {. }
$$

The residuals $e_{\mathrm{i}}$ are assumed to be independently and identically normally distributed, with mean zero and variance $\sigma_{e}^{2}$.

In regression types of analyses, a regression weight expresses the relation between the corresponding predictor and the criterion variable. In this case, the regression weight $\beta$, obtained with ordinary least squares, equals the difference between the sample means, and it is a function of the covariance between the criterion and predictor variables with their predictor values:

$$
\bar{y}_{a}-\bar{y}_{b}=\hat{\beta}=\frac{4}{N} \mathbf{X}^{t} \mathbf{y}
$$

From the perspective of estimation, a possible way for quantifying the information that is obtained is the precision, expressing the certainty around an estimate. Formally, precision represents the expected squared difference between an estimate and the expected estimate. Its inverse is the squared standard error $(S E)$, which provides a scale to use for inferences about that estimate. For the estimated difference between two group means, as just introduced, the $S E$ can be derived using the following equation:

$$
S E_{\beta}=\sqrt{\frac{\sigma_{a}^{2}}{n_{a}}+\frac{\sigma_{b}^{2}}{n_{b}}} .
$$

The $S E$ in this case is a function of the residual variances $\sigma^{2}$ of both groups $a$ and $b$ and their respective number of observations $n_{a}$ and $n_{b}$. Because the residual variances are typically unknown, the $S E$ is estimated using estimates of these variances, based on the samples.

A second way to evaluate the quality of the estimation is bias. Bias is defined as the expected difference between the estimate and the population value. Bias and precision are combined as accuracy, which is formally defined as the inverse of the root mean squared error (RMSE). The RMSE is equal to the square root of the expected $(E)$ squared difference between a population value $(\beta)$ and its estimate $(\hat{\beta})$, and equals the square root of the squared $S E$ plus the squared bias (Kelley \& Maxwell, 2003):

$$
\begin{aligned}
\text { RMSE } & =\sqrt{E\left[(\hat{\beta}-\beta)^{2}\right]} \\
& =\sqrt{E\left[(\hat{\beta}-E[\hat{\beta}])^{2}\right]+(E[\hat{\beta}-\beta])^{2}} .
\end{aligned}
$$

The precision can be used to define a confidence interval for the estimate. Assuming a normal distribution and a nominal Type I error probability $\alpha$ allows for defining a $(1-a) * 100 \%$ confidence interval, which is expected to include the population parameter value with a probability of $(1-a)$. This will only require the $S E$ to scale the standard normal distribution $Z$ that provides the critical values $z$ for the chosen $\alpha$.

$$
\hat{\beta} \pm S E_{\beta} * z_{1-\alpha / 2}
$$

From the perspective of statistical testing, the information is quantified by the statistical power, expressing the probability of correctly rejecting the null hypothesis $H_{0}$ (J. Cohen, 1992). On the basis of a population value $\beta$, its $S E$, and the assumption of normality with nominal Type I error probability $\alpha$, the power for one-sided $t$ tests having sufficient degrees of freedom can be approximated as follows (Hox, 2002; Snijders \& Bosker, 1999):

$$
\frac{\beta}{S E_{\beta}} \approx z_{1-\alpha}+z_{\text {power }},
$$

where $z_{(1-a)}$ and $z_{\text {power }}$ are the values from a standard normal distribution that correspond to the cumulative probability given in the subscripts. The left side of the equation is the noncentrality parameter, such that the power is the proportion of the noncentral $z$ distribution exceeding the critical value $z_{(1-\alpha)}$.

Any of the parameters can be derived as a function of the other parameters. Instead of deriving power given a specific value for the population parameter $\beta$, it can be of interest to derive the number of observations necessary to obtain a certain power. Assuming, for our example, equal residual population variances $\left(\sigma_{\mathrm{a}}^{2}=\sigma_{\mathrm{b}}^{2}=\sigma^{2}\right)$, and equal sample sizes in both groups, the following equation can be derived by combining Equations 3 and 6 :

$$
n \geq 2 \sigma^{2} \frac{\left(z_{1-\alpha}+z_{\text {power }}\right)^{2}}{\beta^{2}} .
$$

Assume, for instance, that group $a$ is expected to score on average at least 1.5 points higher than group $b$, and that there is reason to expect the residuals' standard deviation $(S D) \sigma$ to be 2 , implying an effect size of $.75(=\beta / \sigma)$. To obtain a power of .80 for a one-sided test with nominal $\alpha$ equal to .05 , the required total number of observations is 44 , or 22 in each group.

The equations above require that the residuals from Equation 1 be independent, or they might otherwise lead to an overestimation of the available information, as shown by an underestimation of the $S E$ (Aitkin, Anderson, $\&$ Hinde, 1981). Such wrong estimates of precision would in turn cause statistical tests to be too liberal.

This assumption of independence is violated if observations are clustered, and if the observations within clusters are more alike than observations from different clusters as shown by a positive intracluster correlation $(\rho)$. For a within-cluster variance $\sigma^{2}$ and a between-cluster variance $\sigma_{u}^{2}$, the following equation holds:

$$
\rho=\frac{\sigma_{u}^{2}}{\sigma_{u}^{2}+\sigma^{2}} .
$$


A simple correction for the biased estimate of the $S E$ can be made for situations with groups of exchangeable observations and a constant intracluster correlation, using what is called the design effect (Cochran, 1977). The design effect represents the factor to increase the number of observations $n_{\text {cluster }}$ within each cluster, in order to compensate for the loss of information due to the intracluster correlation $\rho$ :

$$
\text { design effect }=\left(1+\left(n_{\text {cluster }}-1\right) \rho\right) .
$$

Equations 6 and 8 can be combined under strict assumptions of balanced two-level data with equal cluster sizes and only cluster-related differences in the intercept to test for the difference between two groups; for example, a treatment group versus a control group in a clusterrandomized design. Then the above example would, for each of both groups, result in the following equation (Lake, Kammann, Klar, \& Betensky, 2002):

$$
n \geq 2 \sigma_{e}^{2} \frac{\left(z_{1-\alpha}+z_{\text {power }}\right)^{2}}{\beta^{2}}\left[1+\left(n_{\text {cluster }}-1\right) \rho\right] \text {. }
$$

The required number of observations in each group, $n$, is now adjusted for the design effect that is a function of the cluster size and the intracluster correlation. For an intracluster correlation of .25 and a cluster size of 5 , the design effect would be 2 . This causes the required number of observations to be doubled to obtain the same information as for independent observations. In other words, for our example, at least 88 observations are required instead of 44 . Assuming equally sized clusters, 90 observations would be necessary, with 9 clusters of 5 observations assigned to both the experimental group and the control group.

Additional and more elaborated examples can be found in chapter 10 of both Hox (2002) and Snijders and Bosker (1999).

It is noted that the chosen criterion for efficiency can influence the optimality ascribed to a design, because the perspective of power also takes a null hypothesis and a Type I error probability into account, whereas this is not the case in the perspective of accuracy of estimation (Kelley \& Maxwell, 2003).

\section{MULTILEVEL DESIGN EFFICIENCY}

A simple correction for the $S E s$ - for example, using the design effect - often does not suffice because of the very strict assumptions discussed above. Multilevel data analysis provides a flexible alternative. Dependencies among observations are dealt with by modeling them, using clusterspecific effects that are typically assumed to be sampled from normal distributions with variances to be estimated.

\section{Multilevel Model}

The multilevel model is an extension of the ordinary regression model. A criterion variable is regressed on one or more predictors. Yet, according to the multilevel model, the regression coefficients $\beta$ are allowed to differ over groups of observations. If, for instance, the intercept $\beta_{0}$ and one slope $\beta_{1}$ are assumed to be dependent on group membership $j$, the following equation holds:

$$
y_{i j}=\beta_{0 j}+\beta_{1 j} x_{1 j j}+\sum_{h=2}^{p} \beta_{h} x_{h i j}+e_{0 i j}
$$

Although it is possible to estimate a specific intercept and slope for each group $j$, it is often more appropriate to estimate the (co)variances $\sigma^{2}$ of these coefficients over groups, assuming a multivariate normal distribution with zero mean and structured variance-covariance matrix $V$. The random intercept and the random slope can, therefore, be written as follows:

and

$$
\beta_{0 j}=\beta_{0}+u_{0 j}
$$

$$
\beta_{1 j}=\beta_{1}+u_{1 j}
$$

where $u_{0 j}$ and $u_{1 j}$ are normally distributed random variables with parameters

and

$$
E\left(u_{0 j}\right)=E\left(u_{1 j}\right)=0
$$

$$
\begin{aligned}
\operatorname{var}\left(u_{0 j}\right) & =\sigma_{u_{0}}^{2} \\
\operatorname{var}\left(u_{1 j}\right) & =\sigma_{u_{1}}^{2} \\
\operatorname{cov}\left(u_{0 j}, u_{1 j}\right) & =\sigma_{u_{01}}^{2} .
\end{aligned}
$$

The variance and covariance parameters at the second level would result in the following matrix $V_{2}$ :

$$
V_{2}=\left(\begin{array}{cc}
\sigma_{u 0}^{2} & \sigma_{u 01} \\
\sigma_{u 01} & \sigma_{u 1}^{2}
\end{array}\right) \text {. }
$$

The lowest level Equation 11, and the second-level Equations 12 and 13, can be combined (Goldstein, 2003). The residuals are defined at various levels, with the lowest level residuals referred to as $e_{0 i j}$, including an extra index 0 - because it does not depend on any covariates-and $j$, for cluster identification:

$$
y_{i j}=\beta_{0}+\beta_{1} x_{1 i j}+\sum_{h=2}^{p} \beta_{h} x_{h i j}+\left(u_{0 j}+u_{1 j} x_{1 i j}+e_{0 i j}\right) \text {. }
$$

Note that the predictors in the above equation can consist of combinations of other predictors-for example, when dealing with interaction effects or polynomial terms. Furthermore, the predictors in the above equation can be specified at higher levels as well, so that all units within a higher level group share the same predictor value. Finally, the equation shows that the multilevel model consists of fixed as well as random parameters, and can therefore be considered as a special case of the mixed model (Verbeke \& Molenberghs, 2000).

It is possible to further extend this multilevel model by bringing in predictors to explain the random coefficients $\beta_{j}$. For example, a predictor can be included to explain the variance of the intercept over groups, changing Equation 12 to become

$$
\beta_{0 j}=\beta_{00}+\beta_{01} x_{01 j}+u_{0 j} .
$$


Note that Equation 17 would have to be changed accordingly, by bringing in the second-level predictor $x_{01 j}$.

Example: Part one. The model just outlined could fit a study described by Snijders and Bosker (1993), in which a mathematics test is administered to 5 randomly sampled pupils in each of 100 randomly sampled schools for a total of 500 observed test scores. Of primary interest here are the relation between a school's policy and the achievement on the mathematics test $\left(\beta_{3}\right)$ and whether this relation depends on the socioeconomic status (SES) of the pupil $\left(\beta_{2}\right)$, as expressed by the cross-level interaction $\left(\beta_{4}\right)$. IQ is also taken into account, resulting in the following model:

$$
\begin{aligned}
y_{i j}= & \beta_{0}+\beta_{1} * \mathrm{IQ}_{1 i j}+\beta_{2} * \mathrm{SES}_{2 i j}+\beta_{3} \\
& * \text { Policy }_{3 j}+\beta_{4} *\left(\mathrm{SES}_{2 i j} * \text { Policy }_{3 j}\right) \\
& +u_{0 j}+u_{2 j} * \mathrm{SES}_{2 i j}+e_{0 i j} .
\end{aligned}
$$

The test scores are described by a fixed part, including an intercept's coefficient $\left(\beta_{0}\right)$ and a cluster-dependent deviation from that score $\left(u_{0 j}\right)$ for each of the 100 schoolsfor example, Equation 12 , which has a variance $\left(\sigma_{u_{0}}^{2}\right)$ for the random intercept. Each of these group-specific intercepts may also depend on the school's policy of explaining part of its variance (see $\beta_{01}$ in Equation 18).

The relation between the SES and the achievement on the mathematics test may likewise be different for the 100 schools, and may split up in an average relation $\left(\beta_{1}\right)$ and a group-dependent deviation from that relation $\left(u_{1 j}\right)$ (Equation 13), having a variance $\left(\sigma_{u_{1}}^{2}\right)$ for the random slope. Each of these group-dependent relations may also differ according to the school's policy (e.g., $\beta_{4}$ ), explaining part of its variance. The random slope is allowed to covary with the random intercept $\left(b_{u_{0}}\right)$.

Design efficiency parameters. For the multilevel model the design-related parameters include, at each level, the number of units to sample, as constrained by their cost of sampling, and the variance-covariance structure of the random parameters, in addition to the predictor values. The efficiency of the design for estimating or testing parameters further depends on the population values of the model parameters.

As for ordinary regression analyses, increasing the number of observations influences both accuracy and power. This issue is more complex for multilevel data analyses, because each level should have sufficient units sampled to get a fair estimate of the unknown parameters-including the variance-covariance-and enough power for testing them. The number of units that can be sampled at each of the levels is constrained by budget limitations and the costs of sampling these units. Sampling an additional higher level unit (without increasing the total number of lower level units) is typically more expensive than sampling an additional lower level unit from a higher level unit already included in the study, leaving fewer resources for sampling units at the lowest level. If, for example, a decision is made to sample an additional pupil at the lowest level within a newly sampled school at the second level, additional costs could involve contacts with that school and traveling to and from it. A differential cost of sampling units at each level therefore causes a trade-off between having as many higher level units, and as many observations, as possible (M. Cohen, 1998; Mok, 1995; Snijders \& Bosker, 1993).

Increasing the efficiency at one level may reduce the efficiency at other levels (partly because of the trade-off), requiring efficiency to be determined with respect to a certain effect or set of effects. For example, Raudenbush and Liu (2000) note that detecting a cluster $X$ treatment variance is best served by having large enough group sizes, whereas detection of the main effects is enhanced when including more groups, implying smaller group sizes.

Example: Part two. The study in the example above was set up to test for the main effect of a school's policy and its interaction with SES. The formulas to obtain the $S E$ s, derived by Snijders and Bosker (1993), provide a means of comparing this study's design with alternative designs.

Suppose that the costs for including an additional school in the study equal the budget required to observe 5 pupils, reflected by a cost ratio of 5 . With that cost ratio, having 100 schools with 5 pupils in each would require a budget equivalent to observing 1,000 pupils in a single school, instead of only 500. For the same budget and cost ratio, the number of pupils that can be observed in each of 55 (instead of 100) schools would be 13; this means that 715 pupils, instead of 500 , can be observed. If, instead of 55 schools, only 10 are sampled, this results in sampling an average of 95 pupils in each school, for a total of 950 observations. These sample sizes at two levels can be derived using the following equation (Snijders \& Bosker, 1999):

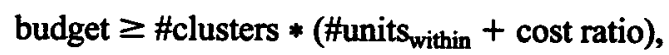

in which the budget is expressed as the number of units that could be observed if all units belonged to the same cluster.

\section{EXPLORING MULTILEVEL DESIGN EFFICIENCY}

Although many researchers are aware of the methodological concerns discussed above, the complexity of the relations involved clearly leads to uncertainty, as illustrated, for instance, by the many questions on optimal allocation on the electronic JSC multilevel modeling discussion list (www.jiscmail.ac.uk/lists/multilevel.html).

Several of these methodological issues have already been addressed, in part analytically and in part by simulation. Both methodologies nevertheless fall short at some point, validating the development of the tool proposed next in this article.

\section{Analytical Methodology}

It is possible to derive $S E$ s and power for estimating and testing specific coefficients of a particular multilevel model, resulting in formulas that provide a quick means to consider a whole range of alternative designs. But the derivation typically requires several simplifying assumptions that restrict the usefulness of the resulting formulas.

A few studies resulted in software capable of performing the calculations to automatically derive the appropriate operational characteristics conditional on a few parameters. Two such tools are briefly discussed. 
A first program, called the power in two-level designs (PINt; Snijders \& Bosker, 1993), is based on large-sample approximations for the $S E$ s in two-level balanced data. These $S E$ s are calculated for each of the regression coefficients, on the basis of a specification of the variances and covariances for the within-cluster covariance matrix and the betweencluster covariance matrix of two-level data. Furthermore, PINt automatically derives conditions to compare, conditional on a budget and costs of sampling units at each of the two levels. As Snijders and Bosker (1993) note, the PINt program cannot be used to derive $S E$ s for the variance components. Furthermore, when having only a few observations or a few groups (fewer than 10), the approximations may be biased (Snijders \& Bosker, 1993).

A second program, called optimal design (OD; Raudenbush \& Liu, 2001), is also based on large-sample approximations. It can be used to compare power for single-level trials, cluster-randomized trials, multisite randomized trials, multisite cluster-randomized trials, cluster-randomized trials with treatment at Level 3, trials with repeated measures, and cluster-randomized trials with repeated measures, providing an easy graphical exploration of the efficiency (Raudenbush, 1997; Raudenbush \& Liu, 2000, 2001). Being based on analytical derivations that rely on restrictive assumptions, this program suffers from limitations similar to PINt's.

Both tools can be used for exploring the basic dynamics between the multilevel data structure and efficiency, considered from the perspective of precision (PINt) or power (OD), ensuring a very quick way to get a lot of insight into the problem of optimal allocation for multilevel data analyses. Furthermore, because estimation of regression coefficients is unbiased when large enough sample sizes at higher levels are involved (Maas \& Hox, 2005), precision is equal to accuracy. For variance estimates there is a bias, and the normality assumption is typically unrealistic.

Unfortunately, many relevant situations have not been dealt with analytically, because relaxing some of the assumptions would immediately complicate the derivation of formulas-for example, when only a few units are sampled at the higher level, or when additional levels are involved. Furthermore, derivations have mainly been restricted so far to the fixed part of the multilevel model, whereas the random part could just as well be of primary interest. For these research situations, it may even be impossible to derive the required formulas in closed form, making it necessary to turn to simulation procedures instead.

\section{Simulation Methodology}

The simulation approach could be an alternative to dealing with the difficulties in trying to establish the efficiency of designs.

A Monte Carlo simulation can be used to build an empirical sampling distribution (ESD) for an estimator as an approximation of the population sampling distribution. This is done by repeatedly analyzing each of a large enough number of data sets that have been simulated conditional on assumed population characteristics (Muthén \& Muthén, 2002). Because data are generated on the basis of chosen population values, the resulting ESD can be used to estimate both accuracy of estimation and power for statistical testing, conditional on the chosen population model. It is possible to construct such a distribution for both estimates, as well as their $S E$ s.

On the basis of the estimates' ESD, the $S E$ can be approximated as the $S D$ of estimates, and the bias can be approximated by the difference between the mean parameter estimate and the population value used for simulation. Power can be approximated as the proportion of replications that leads to a rejection of the null hypothesis. The ESD of the SEs reveals equivalent information, using the $S D$ of estimates as population value. Furthermore, the distribution of estimates can be checked for normality or compared with any other distribution. With a growing number of replications, these approximations will improve, as will the approximation of the ESD they are based on.

Several simulation studies have already been used to explore multilevel designs' efficiency. Although very informative, such simulations only provide ad hoc and nongeneralizable conclusions, since results are conditional on the population characteristics chosen for generating the data, implying that each situation of interest needs to be studied explicitly. Yet, writing research-specific macros to avoid such generalizations often presents too big a challenge for behavioral researchers. This warrants the development of a tool to set up the appropriate macros more easily.

Example: Part three. The main focus of the example so far was on the relation between a certain school policy and the achievements of their students on a mathematics test, possibly mediated by their SES. The model discussed earlier is used for generating possible observations, based on population values for the parameters and the distributions for the predictors and random parameters. Using the same model, the parameters for the predictors for policy and its interaction with SES are consequently estimated, increasingly approximating the sampling distribution with additional replications.

This iterative process can be performed, for instance by assuming 10,55 , or 100 schools in the sample, as discussed above. Each of these three conditions would reveal a sampling distribution allowing for evaluating their efficiency. The ideal condition is that which shows the most precise or accurate estimation, and/or that which shows the most rejections of the null hypothesis, keeping the Type I error probability under control.

\section{ML-DEs}

The ML-DEs tool for exploring the efficiency of multilevel designs is a set of scripts in R ( $R$ Development Core Team, 2004) run sequentially. They allow for setting up macros for simulation and estimation using the specialpurpose multilevel modeling program MLwiN (Rasbash, Browne, Healy, Cameron, \& Charlton, 2005).

\section{Properties of ML-DEs}

The possible problem of complex and error-prone macro specification is circumvented using the freely available and very flexible general-purpose language $R$ to automati- 
cally generate the macros on the basis of a few relevant parameters. This provides not only an easy and flexible tool for programming, but also allows further analyses of the resulting data.

The input for $R$ is a set of parameter specifications, discussed below. A first script (R2MLwiN.R, to be read as "from R to MLwiN") processes these specifications, resulting in several text files that can be executed in MLwiN as macros. Generating these macros has the advantage of clearly bringing forth their structured code, thereby facilitating possible additional changes and promoting an understanding of simulation and macro use in general.

An online form is provided to generate the R-input specifications. The only requirement is an understanding of the parameters involved for multilevel data analyses and their designs.

The MLwiN macros, when executed, result in several tab-delimited text files. These files are prepared for inspection, visualization, and analyses, using a second $R$ script (MLwiN2R.R, to be read as "from MLwiN to R"). Although knowledge of $R$ is not required, it could be useful for further data handling and analyses. The ML-DEs sequence is illustrated in Figure 1.

For each parameter, a text file is stored with the parameter estimates and their estimated SEs over replications, including some basic statistics and information on convergence. As such, they allow for a Wald test for each of the replications, for which the number of rejections of the null hypothesis can be counted. If a likelihood ratio test were requested on any of the random parameters, additional text files would be outputted. Such a test would require a reestimation of the model without the random parameter of interest; for example, leaving out the random slope. The outputted file would contain the unique likelihoods for the full and reduced models in each of the requested likelihood ratio tests. We agree with Self and Liang (1987) that use can be made of a $\chi^{2}$ mixture to interpret the results

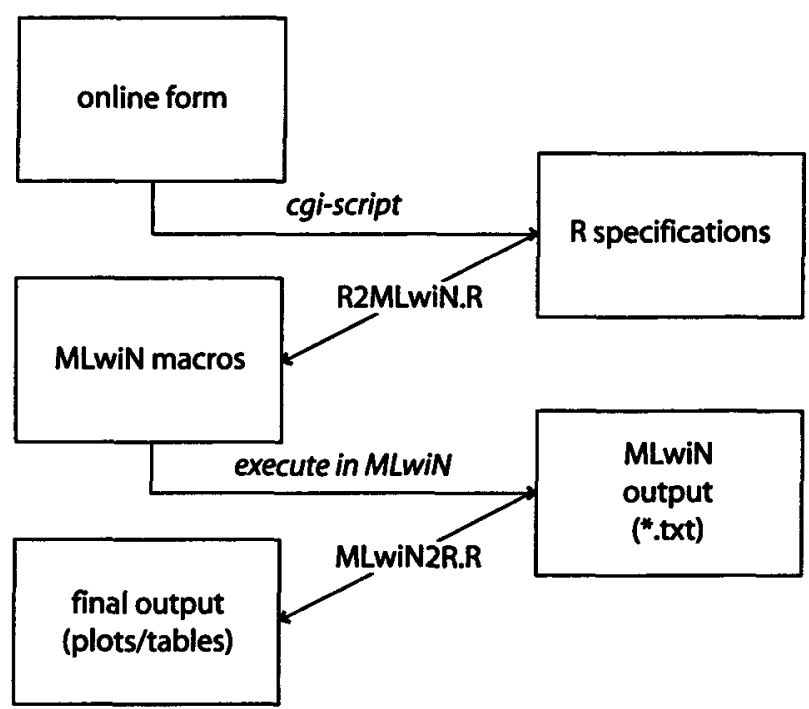

Figure 1. ML-DEs sequence of scripts, starting from enterIng the specifications using the online form toward the plots and tables that result from the MLwiN simulation study. for each of the replications, for which the number of rejections of the null hypothesis can be counted.

The second $R$ script not only reorganizes and summarizes the data, it also specifies functions that can be used for visualization. It allows for plotting the ordered set of estimates and their $S E$ s for each of the conditions, making it possible to compare conditions on sight and taking into account the whole distribution instead of its summary statistic. For the likelihood ratio tests, $p$ values can be plotted for each of the conditions.

The ML-DEs site (ppw.kuleuven.be/cmes/MLDEs .html) can be consulted for additional information and specifications. Some properties of MLwiN are discussed at the MLwiN site (www.cmm.bristol.ac.uk/MLwiN/), from which MLwiN manuals can also be downloaded.

\section{ML-DEs Specifications}

As a further introduction to the properties of ML-DEs, we discuss the required parameter specifications, including four parts: specifications of the model, of the conditions to compare, of the simulation, and of the output. Each ML-DEs simulation study starts with a model specification that is used repeatedly for simulating the data, using population parameter values, in order to compare the conditions of interest, typically differing in sample size at the various levels, by the properties of their ESD.

Model specifications. It is possible to specify up to 30 predictors, each with predictor values assigned to it and linked to a coefficient to express the relation with the (simulated) response.

Because the coefficients partly depend on the predictor values, it is important to be able to specify them in accordance with the expected distribution of predictor values. These values can be specified explicitly, or generated according to a scheme or distribution. The normal distribution is often appropriate, if the predictor values are not known in advance, and are believed to be centered on a mean value with an approximable variance-as is often the case with survey data. Other distributions, such as the Poisson, the binomial, and $\mathrm{chi}^{2}$, are also available. Alternatively, the predictor values sometimes reflect specific conditions - for instance, to imitate experimental conditions or repeated measurements. Several schemes have been implemented for generating such predictors. Finally, several predictors can be combined to specify interactions or polynomial effects.

For assigning group-specific predictor values, the level at which the predictor is defined must be specified. The values are defined in the same way as for the first-level predictors. ML-DEs allows for models with up to five levels. If the online form is avoided, even more levels can be taken into account. All these predictors together define the extended design matrix for fixed parameters. If these predictor values are regenerated for each replication, simulations are based on a population of possible predictor values.

The random parameters include the variances of the residuals at each of the higher levels, which represent the cluster-specific deviations from the mean coefficients. The latter are assumed to have been sampled from a normal distribution with mean zero. For each group-varying 
coefficient - of which there are at most 20 at higher levels - the variance of the residuals must be specified. Predictors with group-specific coefficients must also be defined as each having a fixed part. Covariances can also be specified (default is zero) between random parameters at a certain level. At the lowest level, only one random parameter is allowed, specifying the residual variance at that level. The combination of the specified variances defines the intracluster correlations.

Condition specifications. The conditions that are compared with an ML-DEs simulation study have different sample sizes at the various levels. Two ways of defining these conditions are distinguished in ML-DEs: automatic generating of conditions versus explicit listing of them.

The first way of defining the conditions is to generate them automatically. Conditions are defined on the basis of a specification of the budget, the possible differential cost of sampling units at each of the levels, the range and step size for the number of higher level units, and the maximum number of conditions to be compared. For a two-level model, an example can be found in Snijders and Bosker (1999). Equation 20 is recursively extended to higher levels, each level with its own cost of sampling units. These costs increase for sampling higher level units, further reducing the number of possible observations.

The second way of defining the conditions is to specify them explicitly. Conditions are defined on the basis of a total number of observations rather than on the basis of a budget, and they can differ at each of the levels in their cluster sizes, which need to be specified explicitly. In this way, conditions can be specified very flexibly, as long as they reflect a hierarchical structure and as long as the same total number of observations are implied at each of the levels for all conditions.

Combining the results from different simulation studies easily deals with most limitations in defining the conditions. For example, the automatic generation of conditions requires each study to have conditions differing in the number of groups by a fixed step size. The combination of a first study with cluster sizes 25,50 , and 75 , and a second study with cluster sizes 10,20 , and 30 results in six conditions with the same fixed step size. The same is true when explicitly listing the conditions avoiding the requirement of equal number of observations. An $R$ script is made available to assist in combining the data of different studies.

Simulation specification. Values for the criterion and predictor variables are repeatedly generated, reflecting the variance at each level. For each simulated set of observations, the fixed and random parameters are, consequently, estimated. One such cycle of simulation and estimation is called a replication. Within each replication, an iterative process estimates the fixed part conditional on the random part and vice versa, until convergence, or the maximum number of iterations, is obtained. By default, MLwiN allows for a maximum of 30 such iterations, which may not suffice either because of badly specified models or excessively complex ones. Increasing the maximum number may be a solution to nonconvergence, but processing time will also increase. Replications that do not converge are discarded; the other replications provide the estimates for the parameters.

The resulting empirical sampling distribution serves increasingly well as an approximation with a growing number of replications, but the improvement itself nevertheless decreases. The number of samples or replications should therefore be specified appropriately, depending on the purpose of the analyses. So far, on the basis of a growing stability of the estimates' $S D$, preliminary analyses suggest that about 2,000 should suffice. For exploring the stability, a script that derives a running mean of the $S E$ is included.

A seed can be specified to ensure a specific set of random numbers to be used for generating the predictor values and the random part of the model, permitting an exact replication of the simulation results.

Output specification. The scripts generate output in a prespecified folder, one per study. The output includes text files that serve as MLwiN macros, preliminary results of the MLwiN simulations, and the final R output to use for generating tables and plots. As described above, the final output consists of a list and a summary of the parameter estimates, the corresponding $S E s$, the $\chi^{2}$ value for the variance parameters, and the $p$ values.

An option is to include the actual designs used for simulation, allowing for an inspection of the predictor values, but this option may increase the size of the output files considerably, and will only be of limited use when dealing with questions related to the actual distributions of the predictors in the design matrix.

The count file is interesting during the lengthy simulations to monitor the progress, with information on the current sample and condition. For additional information on the outputted files, we refer to the ML-DEs site (Cools, Van den Noortgate, \& Onghena, 2006).

\section{Example}

ML-DEs can be used to explore the relative efficiency of estimation and testing by comparing the conditions for the model introduced above. Although it is possible to enter and modify the $\mathrm{R}$ specifications in $\mathrm{R}$ directly, it often proves easier to generate them using the online form. Some screen shots are included for illustration in the Appendix.

Specifications. The idea is to compare the efficiency of a design sampling 100 schools with one sampling only 10 schools. The budget specified a maximum of $1,000 \mathrm{ob}-$ servations, and the cost of sampling an additional school was set to be about five times the cost of sampling another pupil to observe in an already sampled school. First the conditions to compare are specified, and the actual twolevel model is specified next.

The study is set up with the three conditions discussed before, with the number of schools being equal to 10 , 55 , or 100 , and with the number of pupils being derived as a function of the budget and cost ratio for sampling schools. On the basis of the chosen budget (allowing up to 1,000 observations), conditions are chosen to be generated automatically. To specify the conditions, they are generated to have a minimum of 10 schools counting upward in steps of 45 to a maximum of 100 . Contrary to 
PINt, distortion of balance is allowed-for example, with group sizes differing (with an $S D$ of approximately 0.5 ). A maximum number of conditions can be specified to avoid ending up with too many conditions to compare, since they all require extensive processing time.

Second, the model is set up with two levels, one for the observed pupils and one for their schools. Policies are assumed to be implemented for the whole school, making it a second-level predictor. IQ and SES are defined at the lowest level. The relation between the SES and the simulated data that represent test scores on a mathematics test is allowed to vary according to school, thereby defining the random slope by allowing it to be random at a second level. The average predicted test scores are also allowed to vary according to school, defining the random intercept, which is specified to vary at the second level as well as at the first, to include the residual variance. Finally, also of primary interestfollowing the example of Snijders and Bosker (1993)-is the interaction between policy and SES, allowing for the relation between policy and the mathematics test score to differ depending on the SES of the pupil. The extended design matrix therefore includes five predictors in total.

Each predictor must be assigned predictor values by sampling them from a normal distribution. Because these predictor values also influence the efficiency of design, they must be chosen in accordance with the population of predictor values for each predictor. PINt assumes a normal distribution for the predictor values; this is often appropriate (e.g., for IQ and SES). This assumption of normality is less obvious for policy, which typically requires a dichotomous variable, indicating whether or not a policy is implemented. Unlike PINt, ML-DEs allows for such dichotomous predictor values, as well as for several distributions, including the normal, binomial, Poisson, uniform, and several others that are discussed in more detail in the ML-DEs manual.

Specifying the predictors as random and allowing them to vary over higher level units requires further specification of their variance at the chosen levels. These values are set up to refer to a lower diagonal variance-covariance matrix, with variances on the diagonal and covariances off diagonal. For the given example, following Snijders and Bosker (1993), the random intercept with variance 0.09, and a random slope with variance 0.0075 , are assumed to covary $(-0.01)$ with a residual variance of about 0.5 .

A nominal significance level $a$ is required for all tests, but alternative $a$ s can easily be considered after simulation, using the outputted estimates. Additionally, the direction of the test is to be specified: left-sided (0), rightsided (1), or two-sided (2).

For the regression coefficients, testing is performed using the Wald test. Contrary to PINt, the variance and covariance parameters can also be considered, as is done with a likelihood ratio test based on the mixture of $\chi^{2}$ (Self \& Liang, 1987). In this case, it is interesting to test for the importance of the random slope for SES; this is carried out by reestimating the parameters with and without the random slope in the model. If several likelihood ratio tests are requested, for each one the original model will be the full model.

The results and all other output for this study will be saved in a prespecified folder, each of which will refer to a particular experiment and will combine all files generated throughout the ML-DEs sequence.

Finally, some simulation specifications must be made. The number of replications is set at 2,000 , to ensure a good enough approximation of the sampling distribution. A seed is chosen to ensure replicability-for the example, we used value one. To reach convergence, the maximum number of iterations is chosen to be 50 .

$R$ code for specifications. The above issues are the only specifications required for setting up a study. This can be done using the online form that results in R-coded specifications. These specifications can be further changed manually, in a text file or directly in R. For the above example, the form's specifications would yield the $R$ code given in Listing 1. Alternative specifications are discussed at the ML-DEs site (Cools et al., 2006).

The estimation and simulation in MLwiN for this example takes approximately $10 \mathrm{~min}$ on an Intel Pentium 4 CPU with $2.80 \mathrm{GHz}, 504 \mathrm{MB}$ of RAM.

Output: Data, tables, and figures. After the appropriate $R$ scripts have been run, the above specifications for the ML-DEs provide a number of output files that are written in the specified folder.

In the first file, the estimates are retained for each of the parameters, including both regression coefficients and (co)variances of random effects. A summary of these estimates is saved in a second file. A third output file is created if likelihood ratio tests are issued, retaining the estimated likelihoods; a fourth file presents the summary of these estimates. Some additional output files are generated to serve the scripts or to provide information on the general setup of the study. Part of the output is discussed next.

For policy (fourth parameter) and its interaction with SES (fifth parameter), the most relevant parts of the output for the conditions with 10 and 100 schools are included in Table 1. Aside from the population effect size, the proportion of rejections, based on the SEs estimated within each iteration, is retained for each of the parameters in each of the conditions ( $\left(\mathrm{emp}_{\text {irical }}\right)$. The $S D$ of the estimates (cfDisp $_{\text {ersion }}$ ) should increasingly reflect the true $S E$, and the proportion of rejections should therefore approximate the nominal alpha for normal distributions. The table additionally provides information on the bias and the population effect, revealing the mean estimates.

Table 1 suggests that the empirical proportion of rejections is higher than the assumed one, as indicated by the nominal $\alpha$ equal to .05 for this example. The difference

Table 1

Summary of the Fourth $\left(\beta_{\text {Policy }}\right)$ and Fifth $\left(\beta_{\text {SES } * \text { Polley }}\right)$ Coefficient for Three Conditions (10/55/100 Schools), Including the Proportion of Rejections, the Number of Valid Samples, the Population Effect Size, the Blas, and Precision

\begin{tabular}{ccccccc}
\hline par & cnd & emp & valid & effect & cfBias & cfDisp \\
\hline 4 & 1 & 0.10096 & 1,971 & 0 & 0.0028 & 0.1194 \\
4 & 2 & 0.05260 & 1,977 & 0 & 0.0008 & 0.0492 \\
4 & 3 & 0.06093 & 1,953 & 0 & -0.0003 & 0.0451 \\
5 & 1 & 0.08574 & 1,971 & 0 & 0.0009 & 0.0431 \\
5 & 2 & 0.05513 & 1,977 & 0 & 0.0002 & 0.0308 \\
5 & 3 & 0.05069 & 1,953 & 0 & 0.0001 & 0.0353 \\
\hline
\end{tabular}


Listing 1

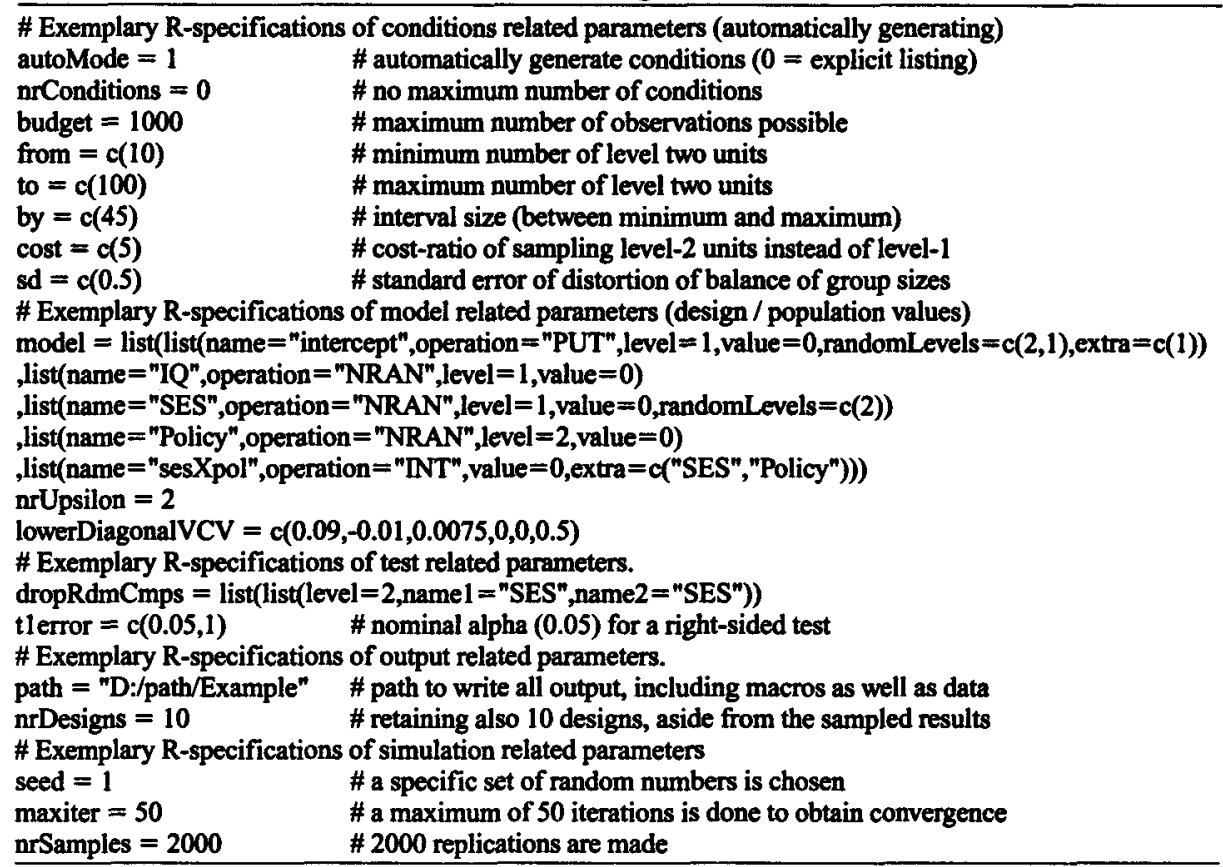

between the empirical and the assumed number of rejections is especially big for the first condition, with only 10 schools sampled. The difference is also somewhat bigger for the fourth parameter (policy), which is defined at the second level, in comparison with the fifth parameter, which refers to the interaction with the first-level, predictor SES. The dispersion of the estimates is clearly much larger for the second-level parameter in the first condition; estimates in that condition, therefore, should not be trusted as much.

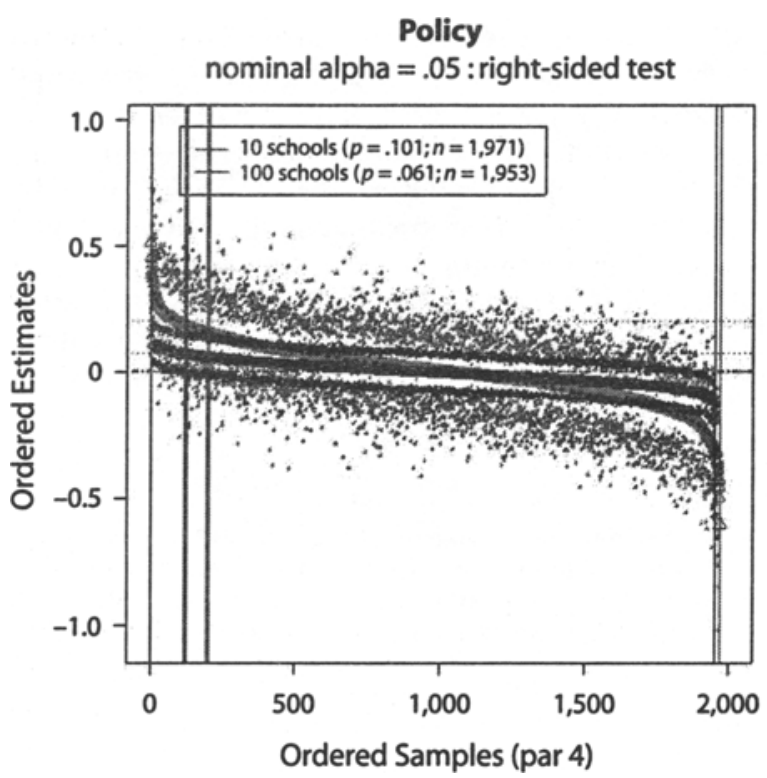

The above results can be visualized using Figure 2 , which is also part of the ML-DEs scripts' output. For each of the two main parameters of interest, a plot shows the ordered set of valid estimates and their confidence intervals for two selected conditions (10/100 schools), revealing their precision.

The precision of each estimate is shown by the upper and lower boundary of its confidence interval, indicated with dots. It can be seen that, for the condition with only 10 schools, the precision of the estimates is generally

Figure 2. Ordered set of valid estimates and their confidence intervals, with the upper and lower boundaries indicated by a dot, for the second-level predictor policy (left panel) and its interaction with the first-level predictor SES (right panel), including vertical lines representing the number of rejections (thick) and number of valid estimations (thin). 
smaller (confidence intervals are larger) and that it varies much more over replications, due to the limited number of units on the basis of which the variance is estimated.

The precision of the estimation can further be approximated by the vertical spread of the estimates (represented by the full curve), showing the condition with only 10 schools to be less precise, although much more so for the second-level predictor policy. The actual number of rejections for a right-sided test is counted over replications, taking a nominal alpha of .05 , and is both shown by a vertical line and included in the legend in addition to the valid number of replications. A horizontal line demarcates quantile $(1-\alpha)$. For samples of only 10 schools, more rejections than expected (based on the nominal $\alpha$ ) are observed. This is even more true of the second-level predictor policy. Both observations are in line with the notion that the SEs, when based on only a few units and/or groups, could be biased when estimated with the programs PINt and OD, because they assume large samples. Simulation studies, as provided by ML-DEs, avoid such biased SEs.

The vertical line on the right indicates the number of valid estimates and can be compared with the 2,000 estimates that were intended, showing a minor loss as a result of nonconvergence. To show potential bias and skewness, additionally, the mean and median can be plotted, but for the regression coefficients, neither bias nor skewness is expected.

For testing the variances at higher levels, the downward bias often becomes severe, and the sampling distributions are only very roughly approximated by a normal distribution, so it is advisable to use a mixture-likelihood ratio test instead of a Wald test. Thus, in addition to the results for the estimates, the results for the likelihoods and their $p$ values are also retained. If a nominal alpha of .05 is chosen, Table 2 shows the number of rejections and some statistics for the distribution of $p$ values that result from the likelihood ratio test with a mixture $\chi^{2}$ distribution.

Again, the results for all the individual estimates can be visualized in the same way as is done for the $p$ values. A nominal alpha of .05 is chosen, resulting in Figure 3, representing the results for all three conditions in the study. For the present specifications, the null hypothesis-that there is no variance of the slope for SES - is rejected in $23 \%-61 \%$ of the replications, depending on the condition under which data were simulated, and is indicated in Figure 3 by the vertical line where the nominal alpha cutoff line does not cross the line representing the $p$ values.

This shows that, given the above specifications, most power is obtained for testing the variance of SES in the

Table 2

Summary of the Likelihood Ratio Test Results for the Random Slopes' (SES) Variance for Each of the Three Conditions (10/55/100 Schools), Including the Number of Rejections, the Population Value for the Random Slopes'Variance, Some Summary Statistics, and the Number of Valid Samples

\begin{tabular}{cccccccc}
\hline cnd & power & valid & effect & plMin & plMd & plMn & plMax \\
\hline 1 & 0.610 & 1,971 & 0.008 & 0 & 0.0773 & 0.2770 & 1 \\
2 & 0.394 & 1,977 & 0.008 & 0 & 0.3099 & 0.4525 & 1 \\
3 & 0.234 & 1,953 & 0.008 & 0 & 0.5897 & 0.5896 & 1 \\
\hline
\end{tabular}

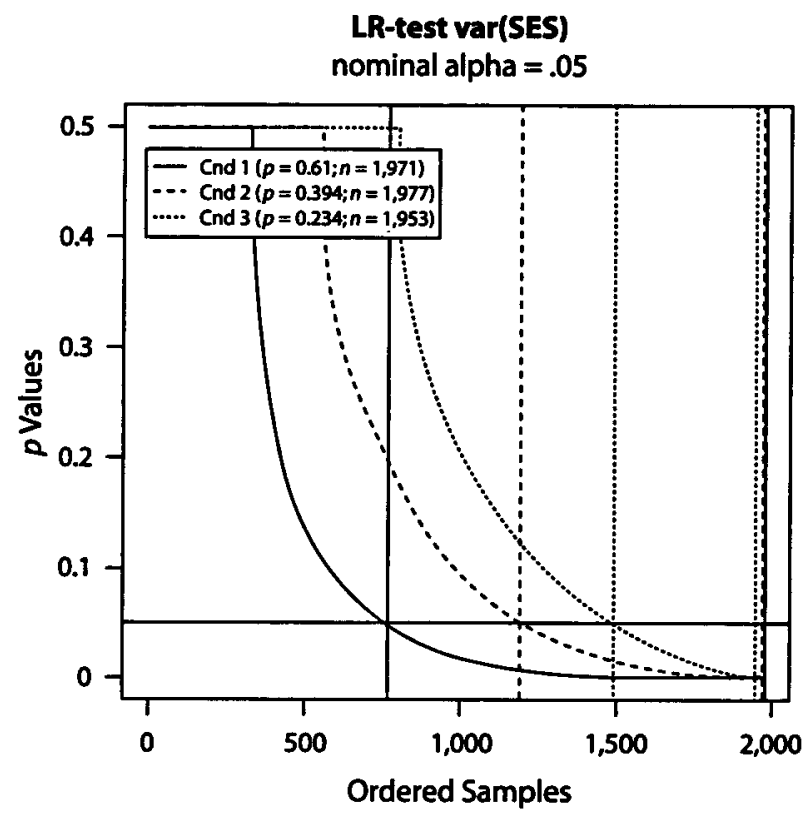

Figure 3. Ordered set of valid estimates of the $p$ value for testing the random slope (SES), for three conditions: 10, 55, or 100 schools sampled. The nominal $a$ (horizontal line) intersects with the number of rejections (vertical line).

first condition, which is the condition with the fewest schools. The plotted $p$ values are derived from an equally weighted mixture of $\chi^{2}$ distributions with 0 and 1 degree of freedom, as before.

\section{CONCLUSION}

Several tools have been developed to assess and compare the efficiency of multilevel designs. These programs are based on analytically derived equations. Although analytical studies do contribute to the general understanding of the relation between the multilevel design and its efficiency, a lot of research conditions cannot be dealt with in this way-for example, with a small sample size at a higher level, with several higher levels, or with the random part of the model.

Simulations often provide an answer, making it possible to study the sampling distribution and its characteristics for virtually any model of interest. The increased processing power of computers today makes this option with considering. But, in general, the results remain indicative and do not allow for straightforward generalization; the use of the simulation studies found in literature can, therefore, often be used only in a tentative way.

The use of ML-DEs assists researchers to set up simulation studies conditional on the specificities of their own research interest, so its results are not dependent on any compromising generalizations.

Especially because results of different studies can be combined, this tool provides strong flexibility. Furthermore, it can provide a first step in programming macros in MLwiN, since it provides structured macros as output that can be further modified to take into account situations not 
incorporated in the tool itself, making it a didactical tool for MLwiN macro programming as well.

For now, the ML-DEs tool only works for continuous outcomes and strictly hierarchical data, but in the future more complex models-including generalized linear mixed models that deal with binary data using PQL and MQL estimation-will be implemented. Also, the inclusion of models for data that are not purely hierarchical is a goal, like cross classification and multiple memberships. Finally, it may prove worthwhile to include alternative schemes for generating predictor values, including correlations between nonnormal predictors.

\section{Availability}

The program ML-DEs consists of a set of scripts and an electronic form (optional). The scripts, the online form and further information on ML-DEs (Cools et al., 2006) can be found at the Web site of the Centre for Methodology of Educational Research at K. U. Leuven: ppw.kuleuven be/cmes/MLDEs.html. The only prerequisite for use of ML-DEs is a citation of this article.

\section{AUTHOR NOTE}

Correspondence concerning this article should be addressed to W. Cools, Katholieke Universiteit Leuven, Vesaliusstraat 2, B3000 Leuven, Belgium (e-mail: wilfried.cools@ped.kuleuven.be).

\section{REFERENCES}

AItKIn, M., ANDERson, D., \& Hinde, J. (1981). Statistical modeling of data on teaching styles. Journal of the Royal Statistical Society A, 144, 419-461.

CoCHRAN, W. (1977). Sampling techniques. New York: Wiley.

COHEN, J. (1988). Statistical power analysis for the behavional sciences (2nd ed.). Hillsdale, NJ: Erlbaum.

COHEN, J. (1992). A power primer. Psychological Bulletin, 112, 155-159.

COHEN, M. (1998). Determining sample sizes for surveys with data analyzed by hierarchical linear models. Journal of Official Statistics, 14, 267-275.

Cools, W., Van den Noortgate, W., \& Onghena, P. (2006). MultiLevel Design Efficiency using simulation (ML-DEs). Retrieved December 24, 2006, from ppw.kuleuven.be/cmes/MLDEs.html.

GoLDSTEIN, H. (2003). Multilevel statistical models (3rd ed.). London: Edward Arnold.

HowELL, D. (2005). Power. In B. S. Everitt \& D. C. Howell (Eds.), Encyclopedia of statistics in behavional science (Vol. 3, pp. 1558-1564). Chichester, U.K.: Wiley.
Hox, J. (2002). Multilevel analysis: Techniques and applications. Mahwah, NJ: Erlbaum.

KeLLEY, K., \& MAXWELL, S. (2003). Sample size for multiple regression: Obtaining regression coefficients that are accurate, not simply significant. Psychological Methods, 8, 305-321.

Lake, S., Kammann, E., KlaR, N., \& Betensky, R. (2002). Sample size re-estimation in cluster randomization trials. Statistics in Medicine, 21, 1337-1350.

MAAs, C., \& Hox, J. (2005). Sufficient sample sizes for multilevel modeling. Methodology, 1, 86-92.

MoK, M. (1995). Sample size requirements for 2-level designs in educational research. Multilevel Modelling Newsletter, 7, 11-15.

Muthén, L. K., \& Muthén, B. (2002). How to use a Monte Carlo study to decide on sample size and determine power. Structural Equation Modeling, 9, 599-620.

PILlemer, D. (1984). Conceptual issues in research synthesis. Journal of Special Education, 18, 28-40.

Rasbash, J., Browne, W., Healy, M., Cameron, B., \& Charlton, C. (2005). MLwiN (Version 2.02) [Computer software and manual]. Retrieved from www.cmm.bristol.ac.uk/MLwiN/.

RAUDENBUSH, S. (1988). Educational applications of hierarchical linear models: A review. Journal of Educational Statistics, 13, 85-116.

RAUDENBUSH, S. (1997). Statistical analysis and optimal design for cluster randomized trials. Psychological Methods, 2, 173-185.

RAUDENBUSH, S., \& LiU, X. (2000). Statistical power and optimal design for multisite randomized trials. Psychological Methods, 5, 199-213.

RAUDENBUSH, S., \& LiU, X. (2001). Effects of study duration, frequency of observation, and sample size on power in studies of group differences in polynomial change. Psychological Methods, 6, 387-401.

R Development Core Team (2004). R: A language and environment for statistical computing. Vienna, Austria: R Project for Statistical Computing Web site: www.r-project.org.

SELF, S. G., \& LIANG, K.-Y. (1987). Asymptotic properties of maximum likelihood estimators and likelihood ratio tests under nonstandard conditions. Journal of the American Statistical Association, 82, 605-610.

SNIJDERS, T. (2005). Power and sample size in multilevel linear models. In B. S. Everitt \& D. C. Howell (Eds.), Encyclopedia of statistics in behavioral science (Vol. 3, pp. 1570-1573). Chichester, U.K.: Wiley.

SNIJDERS, T., \& BOSKER, R. (1993). Standard errors and sample sizes for two-level research. Journal of Educational Statistics, 18, 237-259.

SNUDERS, T., \& BOSKER, R. (1999). Multilevel analysis: An introduction to basic and advanced multilevel modeling. London: Sage.

VAN DEN NoORTGATE, W., \& ONGHENA, P. (2003). Multilevel metaanalysis: A comparison with traditional meta-analytical procedures. Educational \& Psychological Measurement, 63, 765-790.

VAN DEN NOORTGATE, W., \& ONGHENA, P. (2006). Analysing repeated measures data in cognitive research: A comment on regression coefficient analyses. European Journal of Cognitive Psychology, 18, 937-952.

VERBEKE, G., \& MOLENBERGHS, G. (2000). Linear mixed models for longitudinal data. New York: Springer. 


\section{APPENDIX}

The ML-DEs sequence to perform is briefly illustrated, using several documented and "combined" screen shots.

\section{Create Macros}

Specify the parameters in an R window, shown by the R code. Search for the Source R Code of the first script: R2MLwiN.R, and open it. The resulting output should be written as specified by the path, and can now be used to run the macros in MLwiN.

\section{Run Experiment}

In MLwiN, open the macro Run_Experiment.txt from the prespecified folder. Execute the macro using the button at the lower left corner. A possibly lengthy simulation process starts and its results will be retained in the same folder.

\section{Collect Data}

In an $\mathbf{R}$ window, specify the path to locate the data from the study. Search for the Source $\mathbf{R}$ Code of the second script: MLwiN2R.R, and open it. The resulting output should be written as specified by the path and can now be used to visualize and further process the data.

\section{Process the Results}

The second script not only transforms the data to appropriate tables but also defines functions that allow for basic exploration of the data. An exemplary set of functions to use for retrieving and plotting the data is automatically provided. The functions require arguments that are first defined by means of example. A first set of functions is used to retrieve the information on the study, the estimates, their summary statistics, possible likelihoods and, finally, their summary statistics. A second set of functions is used to visualize the individual estimates, standard errors, and likelihoods and their summary statistics. For any later use of the data, an alternative script is provided that omits the reorganization of data that therefore become obsolete.

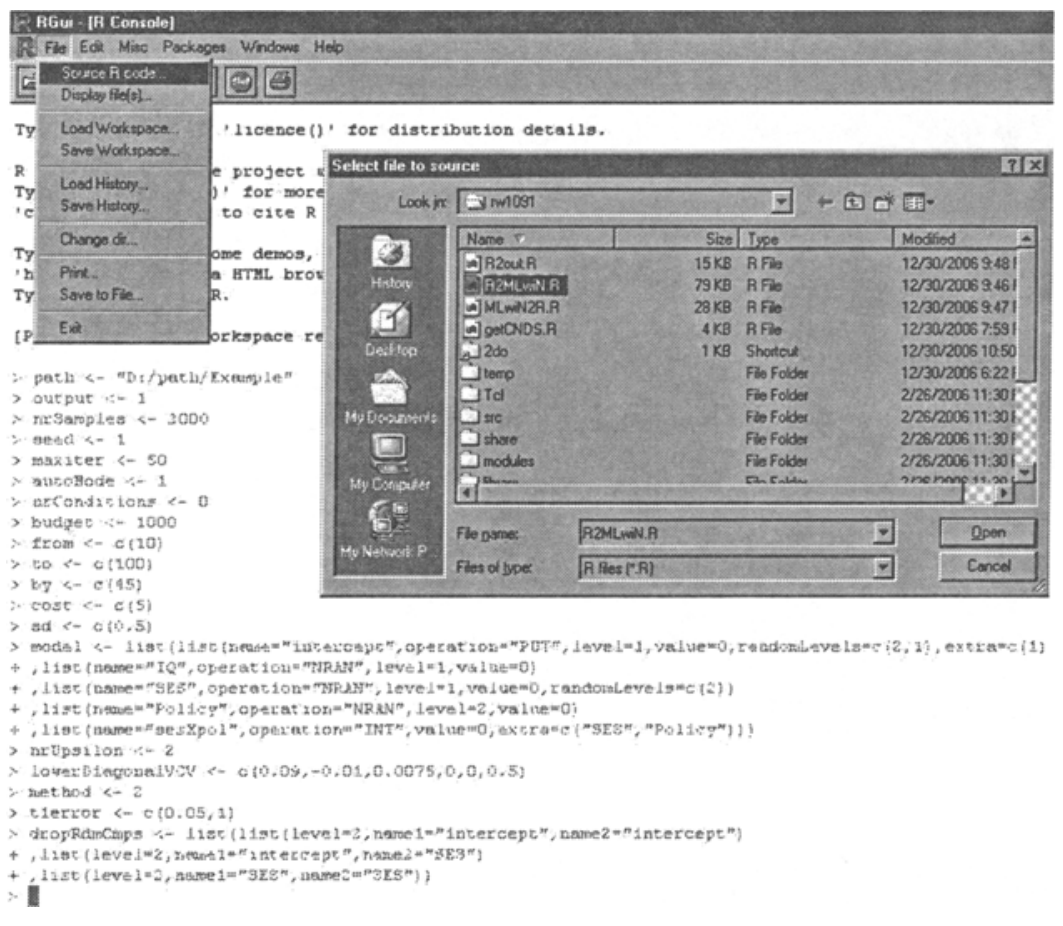

Figure A1. Create MLwiN macros based on the specifications using the R2MLwiN.R script. 


\section{APPENDIX (Continued)}

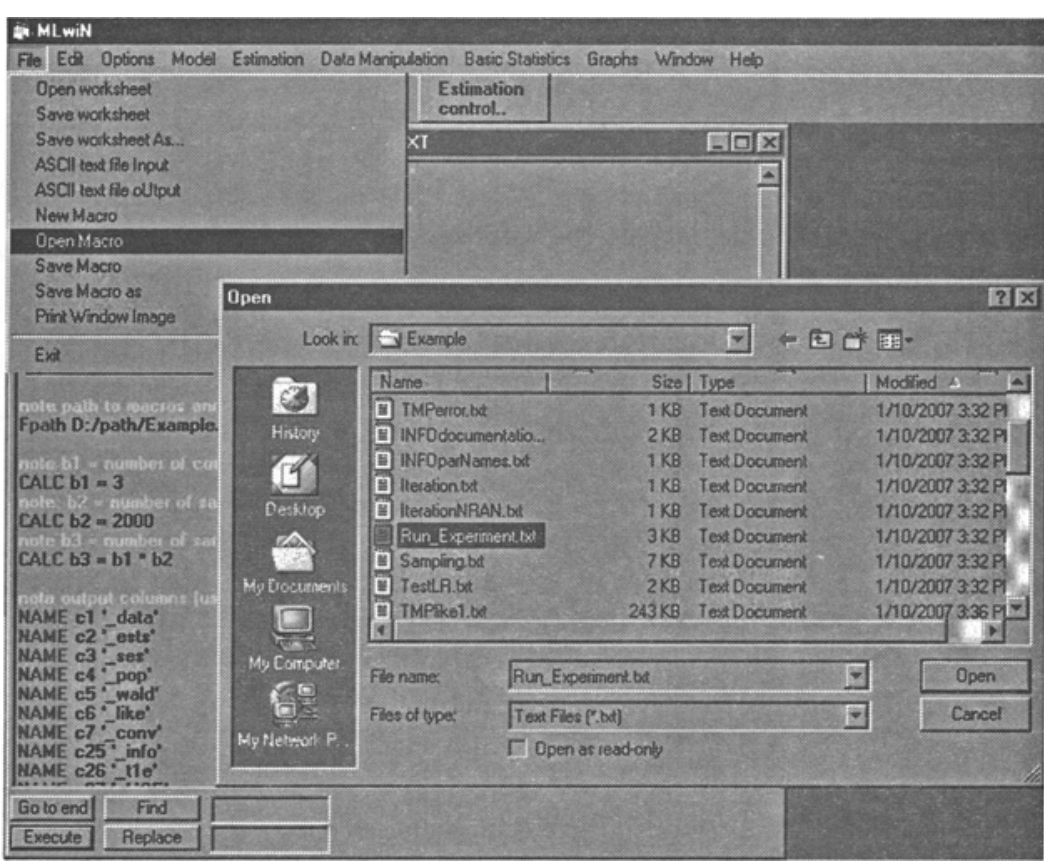

Figure A2. Run experiment from within MLwiN, opening the MLwiN macro file Run_Experiment.txt and executing it.

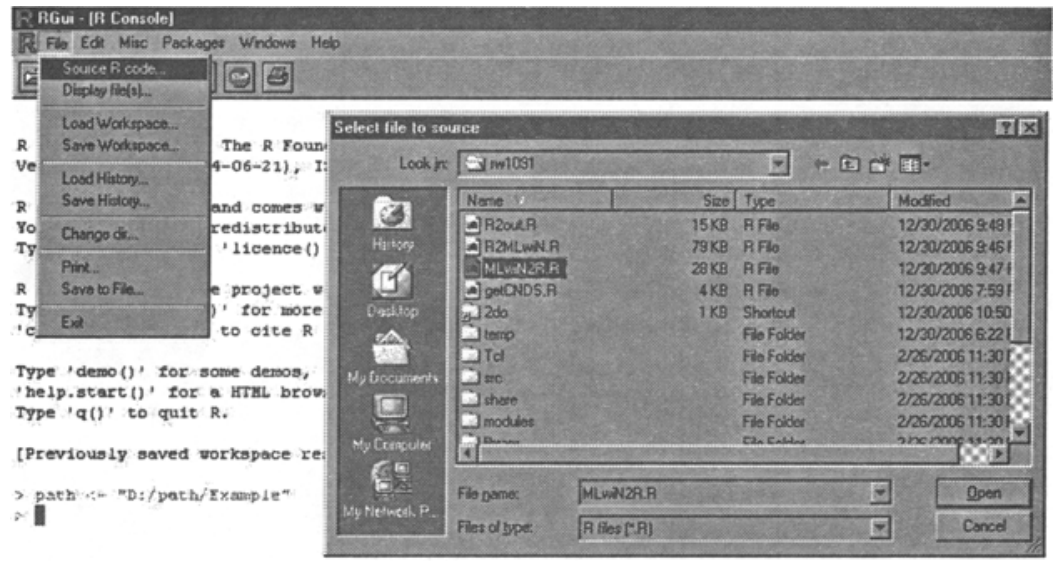

Figure A3. Collect and handle the MLwiN macros' output using the MLwiN2R.R script for the data in the appropriate folder. 


\section{APPENDIX (Continued)}

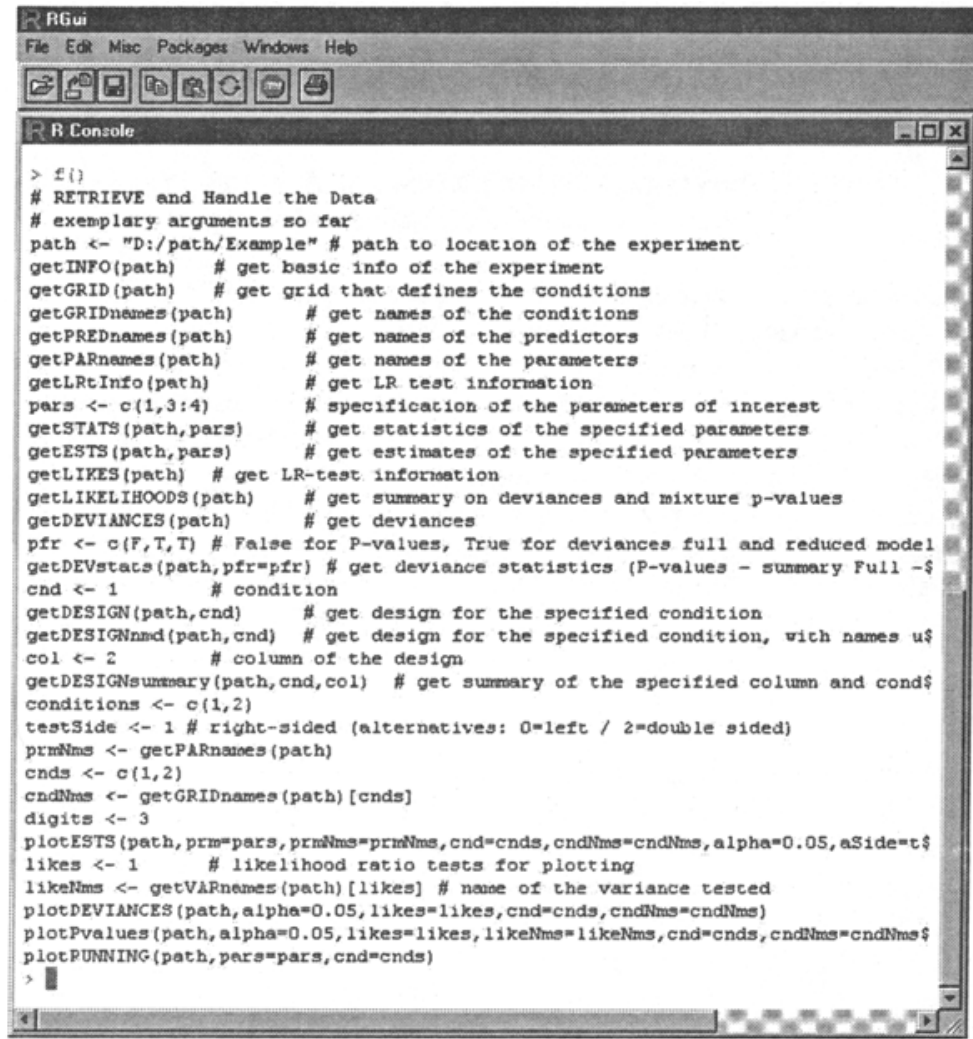

Figure A4. Functions that are included in ML-DEs are exemplified and can be retrieved using the code $\mathrm{f}(\mathrm{O}$ after MLwiN2R.R has been run.

(Manuscript received January 26, 2007;

revision accepted for publication March 28, 2007.) 